\title{
Optical and Chemical Effects Governing Femtosecond Laser-Induced Structure Formation on Polymer Surfaces
}

Youssef Assaf $^{a}$, Anne-Marie Kietziga*

${ }^{a}$ Department of Chemical Engineering, McGill University, 3610 University Street, Montreal, Canada, H3A OC5

Youssef Assaf: +1(514) 946-8893; youssef.assaf@mail.mcgill.ca

*Anne Kietzig (corresponding author): +1(514) 398-3302; anne.kietzig@ mcgill.ca 


\begin{abstract}
With the emergence of femtosecond technology, laser machining has recently led to the creation of novel porous structures on polymers. However, the mechanism behind their formation is yet to be understood. In this study, the dependence of femtosecond laser-induced surface structure on processing parameters is established at two distinct wavelengths (800 nm and $275 \mathrm{~nm}$ ) for six different polymer films: LDPE, PC, PET, PLA, PMMA, and PTFE. All of the observed structures are then optically and chemically characterized as a first step towards elucidating their formation mechanism. The threshold fluence at operating conditions was determined to be the main parameter affecting porosity formation during machining. Furthermore, for transparent films, a transition from multiphoton to linear absorption is observed to occur at $800 \mathrm{~nm}$ but not at 275 $\mathrm{nm}$. This shift in optical properties was determined to be a major contributor to incubation effects. These observations are also in agreement with UV/VIS analysis as measurements show that polymers with a cut-off wavelength lower than that of the laser beam undergo a shift in absorption behavior. Finally, some polymers experience a continuous darkening of their surface with increasing fluence due to an increasing degree of photo-oxidation.
\end{abstract}

\title{
Keywords
}

Laser micromachining; polymer; absorption mechanism; photochemical reaction, incubation

\section{INTRODUCTION}

Laser micromachining is a single-step, contactless method to alter the structure and properties of a material's surface [1]. With the emergence of femtosecond (fs) laser technology, this technique now allows the impartation of hierarchical roughness to target surfaces down to the nano-scale [2]. This sets it apart from other surface texturing techniques like etching and lithography since any micro-scale pattern that is inscribed into the surface through material ablation will further exhibit nanometric features without the need for an additional processing step [3]. In addition to such laser-inscribed structures, subjecting a surface to femtosecond laser radiation can lead to self-assembled structures with dimensions smaller than the focal spot size [4]. Such laser-induced microstructures have been extensively studied on metals due to their unique topography. For example, laser-induced periodic surface structures (or LIPSS) and hierarchical topologies can be used for the generation of structural color [5] or superhydrophobic surfaces [6- 
8] respectively. Thus, through the creation of unique laser-induced structures, fs laser micromachining now allows the development of engineered metal surfaces with completely novel properties. In contrast, very few studies describe the production of laser-induced surface structures on polymers. Some notable examples include Baudach et al.'s (2000) observation of bubble formation on PC and PMMA after machining [9] and Yada et al.'s (2015) study of LIPSS formation on PLA [10]. The aim of this study is therefore to characterize the different laserinduced microstructures that appear on polymer surfaces under different machining conditions as a first step towards elucidating their formation mechanism. The polymers chosen for this study were films of: low density poly(ethylene) (LDPE), poly(lactic acid) (PLA), poly(carbonate) (PC), poly(ethylene terephthalate) (PET), poly(methyl methacrylate) (PMMA), and poly(tetrafluoroethylene) (PTFE). In addition to their wide commercial availability, they contain a variety of chemical functional groups that will allow a better identification of the effect of chemical composition on machining results. Finally, their respective optical properties are varied enough so that the effect of the latter on machining results can be determined.

The most commonly reported laser-induced structures on polymers are LIPSS whose orientation is dependent on the incoming light polarization [11, 12]. Another feature of interest is laser-induced porosity, which has been observed to appear on PMMA [13, 14] and PC [14]. This phenomenon was first described by Baudach et al. (2000) and was attributed to bubble formation during melt re-solidification. The aforementioned studies were mostly interested in fundamental laser-polymer interaction and therefore only described local structure formation on the bottom or side-walls of ablated craters as opposed to texturing an area larger than the focal spot size. Recently, Liang et al. (2014) have demonstrated that homogeneously texturing poly(tetrafluoroethylene) (PTFE) leads to the formation of an air-trapping porous structure that is effectively superhydrophobic [15]. Since then, our recent work has shown that similar structures can form on a wide variety of polymers [16]. Such structures, that are both porous and exhibit a dual-scale roughness, are prime candidates for applications where a large surface area is required; especially in the biomedical field where these properties can enhance cell adhesion and growth on scaffolds and grafts. Indeed Martinez et al. (2011) reviewed how enhancing surface roughness can lead to the increased endothelialisation of stents [17]. Furthermore, in their review, Rashid et al. (2004) state that microporosity in synthetic grafts can help increase healing and angiogenesis [18]. However, the properties of such high surface area structures as well as their formation mechanism 
is still unknown. Due to the complex nature of laser-polymer interactions, these novel structures are expected to have properties that are significantly different from the base material because of the defects generated during laser irradiation. These defects might have a physical origin due to a local increase in absorption [19] or a chemical origin due to preferential bond breaking [20]. Literature suggests that such phenomena might also be highly dependent on wavelength [21] or photochemical reactions that occur during machining [22-24]. The sum of these processes makes polymers very sensitive to pulse number. This dependence is generally termed as incubation effects and was first observed to occur for a polymer in Baudach et al'.s study (1999) of poly(imide) ablation [25]. In summary, optical and chemical properties are essential parameters that not only dictate polymer machining behavior, but also change during the process. Tracking the evolution of these properties as a function of machining conditions will therefore offer substantial insight into the mechanisms at play. Such a comprehensive optical and chemical characterization of fs laser-induced structures on a variety of polymers will therefore give significant insight into their formation mechanisms. This improved understanding of the process will later allow the optimization of machining conditions in order to obtain structures with specific physico-chemical properties.

Our study therefore focuses on machining various polymers at two different wavelengths: $\lambda=275 \mathrm{~nm}$ and $\lambda=800 \mathrm{~nm}$. These values were chosen because most of the studied polymers have their cut-off wavelength located in between these two values thus allowing us to determine the effect of band gap value on machining. The resulting structures were characterized by measuring their transmittance, reflectivity, chemical composition, and bonding structure. The threshold fluence and incubation coefficient of all materials were also measured at both wavelengths in order to observe if they are correlated to any of the aforementioned properties. All of the abovementioned measurements were then related to absorption mechanisms and photochemical pathways in order to determine the processes governing the formation of fs laser-induced structures on polymer surfaces. 


\section{MATERIALS AND METHODS}

\subsection{Materials:}

For each polymer, $3 \times 10 \mathrm{~cm}$ samples were cut from films with different thicknesses: $50 \mu \mathrm{m}$ for PMMA (Evonik Industries) and LDPE (3Spack laboratory), $75 \mu \mathrm{m}$ for PLA (BI-AX International Inc.), and $100 \mu \mathrm{m}$ for the rest (McMaster-Carr).

\subsection{Laser micromachining:}

Samples were machined using a Ti:Sapphire laser (Coherent Libra) delivering a Gaussian beam at a wavelength of $800 \mathrm{~nm}$ and in pulses of duration $\tau=85 \mathrm{fs}$ at a repetition $f_{p}$ of $1 \mathrm{kHz}$ (Table 1). For operation at $800 \mathrm{~nm}$, the beam power was attenuated down to the desired operating power $P$ through a digitally controlled half-wave plate and a polarizing beam splitter. The beam was then focused through a $100 \mathrm{~mm}$ lens down to a $44 \mu \mathrm{m}$ spot size $\omega_{0}$ onto the sample whose surface lied at the focal plane. The value of $\omega_{0}$ was obtained by machining lines and fitting their measured widths to Liu et al.'s (1982) model [26]. The sample was placed on a digitally controlled stage (Newport corporation), whose trajectory and velocity $v$ were controlled through the GOL3D software (GBC\&S). For operation at $275 \mathrm{~nm}$, the beam was passed through an optical parametric amplifier (Coherent Opera Solo) where the desired wavelength was produced. Similarly, the beam was attenuated through a variable neutral density filter (Newport corporation) before being focused through a $175 \mathrm{~mm}$ lens down to a $35 \mu \mathrm{m}$ spot size. For this beam path, the sample was also machined at focus and positioned on another motorized stage (Zaber Technologies Inc.). For single pulse ablation, the beam was further passed through a selective beam chopper that effectively reduced the beam repetition rate down to $25 \mathrm{~Hz}$. Finally, all samples were subjected to a five minute ethanol sonication bath immediately after machining in order to get rid of any loose machining debris. 
Table 1: Laser characteristics

\begin{tabular}{|c|c|c|c|c|c|c|}
\hline Gain medium & Profile & $\lambda(\mathbf{n m})$ & $\tau(\mathbf{f s})$ & $f_{p}(\mathbf{k H z})$ & $\begin{array}{c}\omega_{0}(\mu \mathrm{m}) \text { for } \\
\lambda=800 \mathrm{~nm}\end{array}$ & $\begin{array}{c}\omega_{0}(\mu \mathrm{m}) \text { for } \\
\lambda=275 \mathrm{~nm}\end{array}$ \\
\hline Ti:sapphire & Gaussian & $800 / 275$ & 85 & 1 & 44 & 35 \\
\hline
\end{tabular}

Laser-induced structures were created by raster scanning the sample at different single pulse peak fluences $F_{0}$ (equation 1) and at a pulses-per-spot number PPS of 55 (equation 2) over a square area with dimensions $14 \times 14 \mathrm{~mm}$. The spatial overlap $\Phi$ between two consecutive lines in the raster scan was kept fixed at 0.95 in order to ensure a homogeneous structure.

$$
\begin{aligned}
& F_{0}=\frac{8 P}{\pi \omega_{0}^{2} f_{p}} \\
& P P S=\frac{\omega_{0} f_{p}}{v(1-\phi)}
\end{aligned}
$$

As previously mentioned, due to incubation effects, the threshold fluence $F_{\text {th }}$ is a function of PPS. The magnitude of this dependence is dictated by the material-specific parameter $\xi$, which is termed as the incubation coefficient. The single pulse threshold fluence $F_{t h}(1)$ and $\xi$ were obtained for each material by machining lines at different $F_{0}$ (equation 1) and PPS (equation 2 with $\Phi$ set to 0$)$. At each PPS, the logarithmic dependence of the depth-per-pulse $d$ on $F_{0}$ was then fit to equation 3 in order to obtain $F_{t h}$ and the effective absorption coefficient $\alpha_{\text {eff }}$ of the material.

$$
d=\frac{1}{\alpha_{e f f}} \ln \left(\frac{F_{0}}{F_{t h}}\right)
$$

$F_{t h}(1)$ and $\xi$ are finally calculated by fitting the dependence of $F_{t h}$ on PPS according to the incubation model developed by Jee et al. (1988) (equation 4) [27].

$$
F_{t h}(P P S)=F_{t h}(1) P P S^{\xi-1}
$$




\subsection{Topographical characterization:}

Structures were imaged by scanning electron microscopy (SEM) (FEI Inspect F50) by sputter coating the samples with a $10 \mathrm{~nm}$ layer of gold in order to avoid surface charging. For the threshold determination experiments, ablation depths were measured by 3D confocal microscopy (Olympus LEXT OLS4000).

\subsection{Optical characterization:}

Optical characterization of the various films was performed with ultraviolet-visible spectrophotometry (UV-VIS) in transmittance mode (Thermoscientific Evolution 300). The instrument was fitted with an absorbent screen containing a circular aperture ( $3 \mathrm{~mm}$ diameter) to ensure that the incoming light is only passing through the machined area of interest. The $100 \%$ transmittance baseline was measured in air with the aperture installed. Reflectance measurements were performed with an integrating sphere accessory (Cary 100) internally coated with barium sulfate paint and using Spectralon ${ }^{\circledR}$ standard disks (Labsphere) for the $100 \%$ reflectivity baseline. An integration time of 15 seconds was used for each measurement in order to minimize the effect of noise.

\subsection{Chemical characterization:}

Chemical characterization of the various films was performed by X-ray photoelectron spectroscopy (XPS) (Thermoscientific K-alpha). Measurements were taken using a $400 \mu \mathrm{m}$ spot size with an Argon ion flood gun turned on in order to compensate for surface charging. Two types of scans were performed for each sample. A survey scan ( $200 \mathrm{eV}$ pass energy, 3 scans, $10 \mathrm{~ms}$ dwell time) in order to determine composition and high resolution scans (50 eV pass energy, 10 scans, $50 \mathrm{~ms}$ dwell time) for the 1s sublevel of Carbon, Oxygen, and Fluorine (PTFE only) in order to determine bonding structure. Peak allocation for the base materials was based on Beamson and Briggs' database [28]. Therefore, the same background subtraction method (linear) and peak fitting function (Gaussian-Lorentzian sum) were used. All samples were stored in a vacuum oven set at $30^{\circ} \mathrm{C}$ prior to analysis to ensure their dryness. 


\section{RESULTS AND DISCUSSION}

\subsection{Initial observations:}

Figure 1 illustrates the different laser-induced structures obtained at $800 \mathrm{~nm}$ as well as the color change associated with their appearance. For all materials, no ablation was observed below $F_{0}=1.3 \mathrm{~J} / \mathrm{cm}^{2}$. Above that fluence, a porous structure appears on all polymers which makes them lose their transparency and gives them a light color ranging from white (PTFE, PLA) to light brown (PC). For PC and PET, the structure seems to form nanoparticle clusters at higher fluences and, in the case of PC, eventually leads to a loss in porosity. LDPE structure seems to be affected as well since the structure bundles up as fluence increases. Looking at the sample colors, we can see that all three of these polymers undergo a progressive darkening of their surface, with PC eventually becoming black. Furthermore, these three polymers are the only ones who experience a darkening before the ablation threshold is reached. Indeed, at $F_{0}=0.6 \mathrm{~J} / \mathrm{cm}^{2}$, a darkening is observed even though no structure has appeared yet. Finally, PLA, PMMA, and PTFE had both their color and structure unaffected by increasing fluence. 


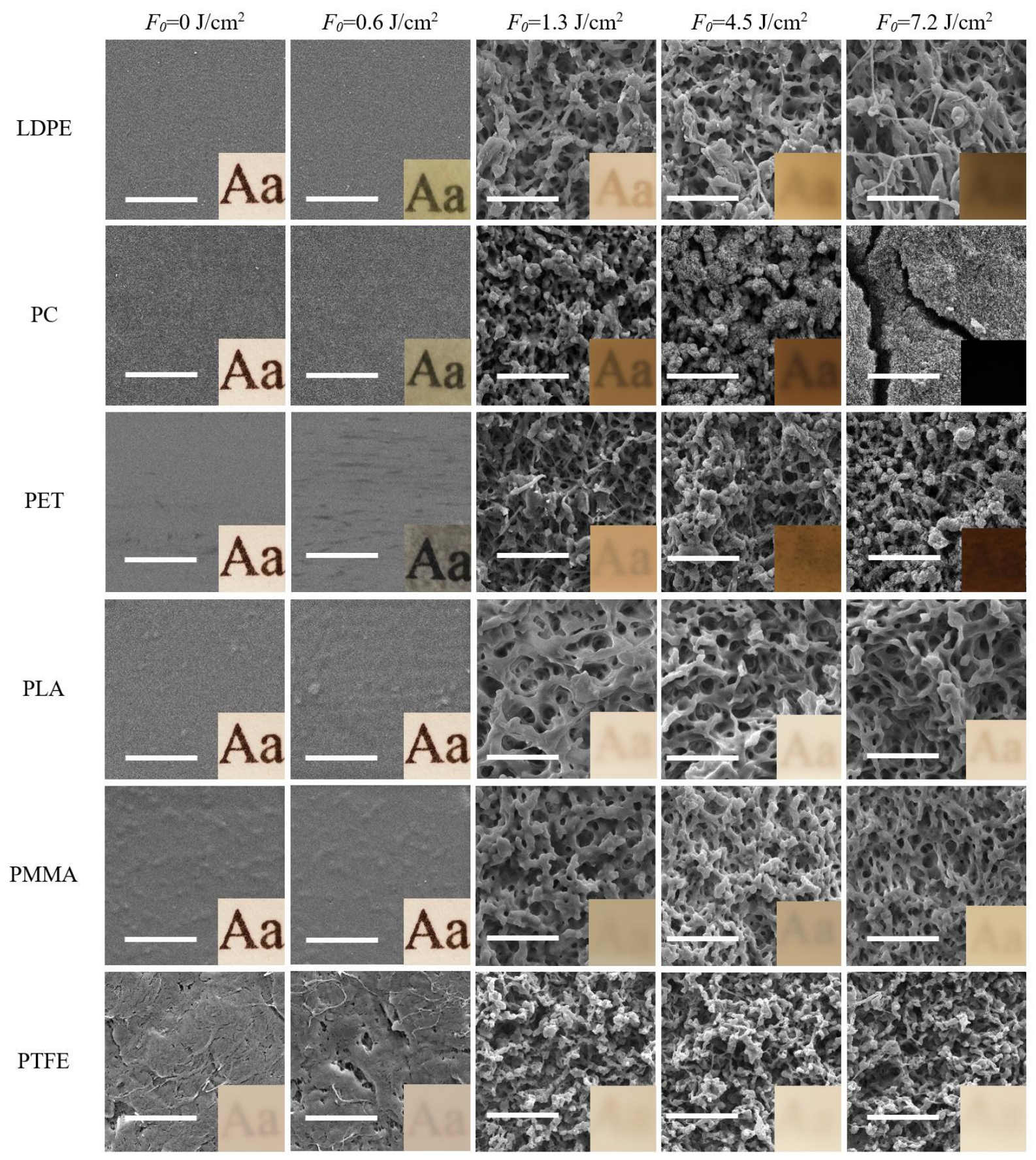

Figure 1: SEMs of laser induced microstructures at different fluences for a wavelength of $800 \mathrm{~nm}$. All scale bars represent $4 \mu \mathrm{m}$. Inset is a $3 \times 3 \mathrm{~mm}$ photograph of the sample placed on white paper on which letters were printed to highlight the change in transparency. 


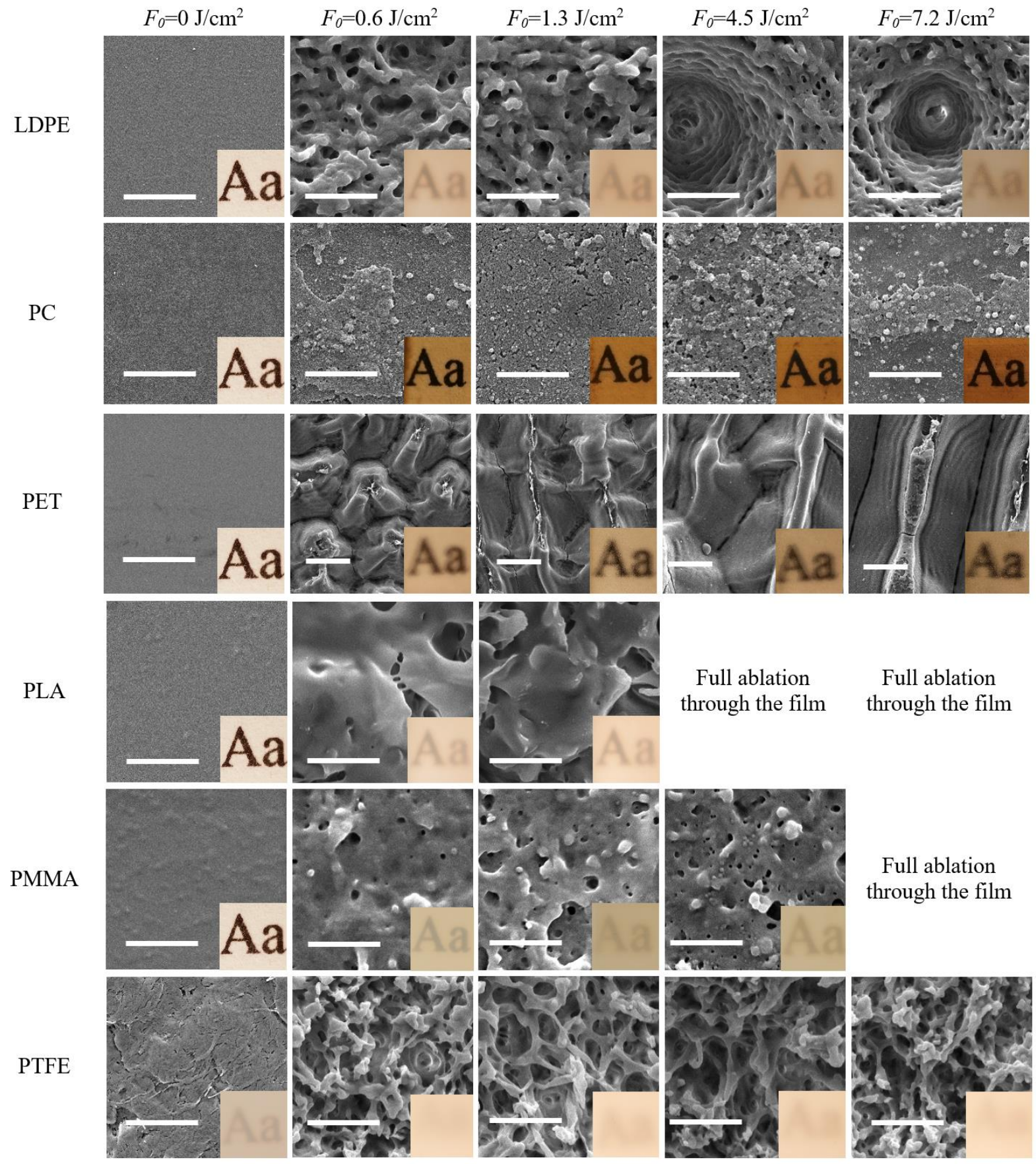

Figure 2: SEMs of laser induced microstructures at different fluences for a wavelength of $275 \mathrm{~nm}$. All scale bars represent $4 \mu \mathrm{m}$. Inset is a $3 \times 3 \mathrm{~mm}$ photograph of the sample placed on white paper on which letters were printed to highlight the change in transparency. 
As illustrated in Figure 2, machining at a wavelength of $275 \mathrm{~nm}$ resulted in significantly different microstructures. First of all, for all polymers, structures appeared at a lower fluence than at $800 \mathrm{~nm}$ indicating a decrease in threshold fluence. PTFE is the only material that exhibited a similar porous microstructure and color at both wavelengths. While a porous structure still formed for LDPE, new circular features appeared during UV ablation. As seen on the SEMs, starting at $F_{0}=4.5 \mathrm{~J} / \mathrm{cm}^{2}$, certain regions of the surface underwent increased ablation with circular craters of around $4 \mu \mathrm{m}$ starting to appear in between the typical porous structure. For the rest of the materials, the surface exhibits significant melt with limited porosity. Some nanoparticle cluster formation is observed on PC while the structures on PET looks like melt bumps that transition into grooves at higher fluence. The limited microstructure formation is also reflected in the color of the samples as they retain their transparency to different degrees indicating that only a shallow melt layer remained after ablation. Overall, visual inspection of the samples indicated that a significant change in optical properties was occurring during machining. So, as a first step, UV/VIS spectrometry was performed on all samples in order to track the evolution of those properties as a function of machining parameters.

\subsection{Optical characterization:}

For all materials, a very large decrease in transmittance was observed when the ablation threshold was reached. Figure 3 exemplarily presents the resulting spectra for PC. 


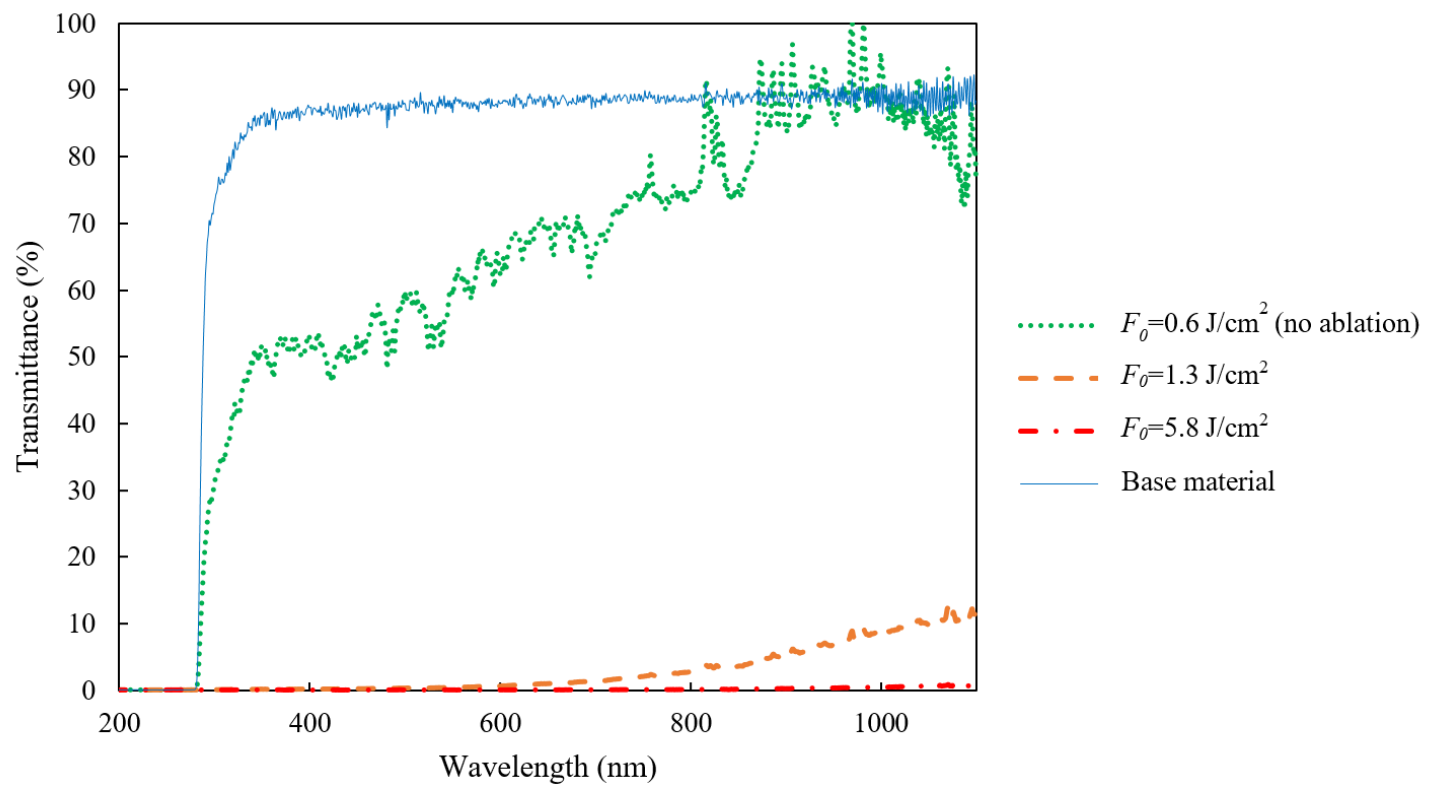

Figure 3: Evolution of the transmittance spectrum of PC as a function of fluence.

The transmittance spectrum for non-machined PC is typical of a dielectric material. The material is fully absorbent below its cut-off wavelength $\lambda_{c}=281 \mathrm{~nm}$ while it transmits almost all of the incoming light above that value. The optical bandgap energy of the material therefore corresponds to the energy of a photon having a wavelength of $\lambda_{c}$. The number of photons that need to be absorbed simultaneously in order to overcome that bandgap at any given wavelength $\lambda$ is therefore equal to the rounded-up ratio of $\lambda$ and $\lambda_{c}$. This value is thus equivalent to the order of the dominating multiphoton absorption process $m$. In the case of PC, for a wavelength $\lambda=800 \mathrm{~nm}$, this corresponds to $m=3$ and a 3 -photon absorption process. However, Figure 3 clearly indicates a shift in absorption behavior with increasing machining fluence. Indeed, the original step-like spectrum gradually shifts until almost reaching a completely flat line at $F_{0}=5.8 \mathrm{~J} / \mathrm{cm}^{2}$. The latter is typical of absorbing materials. This is clear indication that the optical properties of the formed microstructure are very different from those of the original material.

Figure 4 summarizes both the transmittance and the reflectance data at $\lambda=800 \mathrm{~nm}$ and $\lambda=275 \mathrm{~nm}$ for the polymers machined at the respective wavelengths. At $\lambda=800 \mathrm{~nm}$, for all materials, 
a significant increase in reflectance is observed when the threshold for ablation is reached $\left(F_{0}=1.3\right.$ $\mathrm{J} / \mathrm{cm}^{2}$ ). As can be seen in Figure 1, this corresponds to the appearance of the porous structure. Its roughness is therefore enhancing the scattering and diffuse reflection of all incoming visible wavelengths giving most of the films a white appearance. At the same time, an even larger decrease in transmittance is observed. This confirms the fact that the laser-induced structures, unlike the transparent original materials, are actually absorbent with respect to the machining wavelength. While these observations apply to all polymers, two main groups can be distinguished. For PLA, PMMA, and PTFE, the optical properties do not seem to be affected by increasing fluence beyond the ablation threshold as their reflectance and transmittance remain somewhat constant. On the other hand, for LDPE, PET, and PC, reflectance and transmittance are continuously decreasing with fluence. This indicates that, not only are the laser-induced structures more absorbent that the original material, they are also becoming more and more absorbent as fluence increases. Furthermore, unlike the previous three polymers, these materials also experience a significant drop in transmittance before the ablation threshold is reached $\left(F_{0}=0.6 \mathrm{~J} / \mathrm{cm}^{2}\right)$. This phenomenon can be observed in Figure 1, where the films exhibit some darkening even before the microstructure is formed. Figure 3 also already indicated such behavior as the spectrum corresponding to irradiation at $F_{0}=0.6 \mathrm{~J} / \mathrm{cm}^{2}$ shows a slight decrease in transmittance compared to that of the pristine material. This could be due to the fact that randomly distributed physical defects are being generated by the irradiation that locally enhance the optical field. Nagilhou et al. (2015) [29] recently presented data that supports that notion for poly(styrene) in their extension of the defect model [30]. However, the fact that this phenomenon was only observed on some of the polymers and that it was accompanied with some sort of coloration could also indicate that photochemical reactions are taking place. For $\lambda=275 \mathrm{~nm}$, polymers with a cut-off wavelength larger than $\lambda$ (values can be found in Table 2) had negligible transmittance and reflectance at all fluences since they absorb almost all of the incoming light at this wavelength. The two exceptions, LDPE and PLA, are still transparent to the machining wavelength before irradiation. However, this transparency is quickly lost when the material is ablated. 

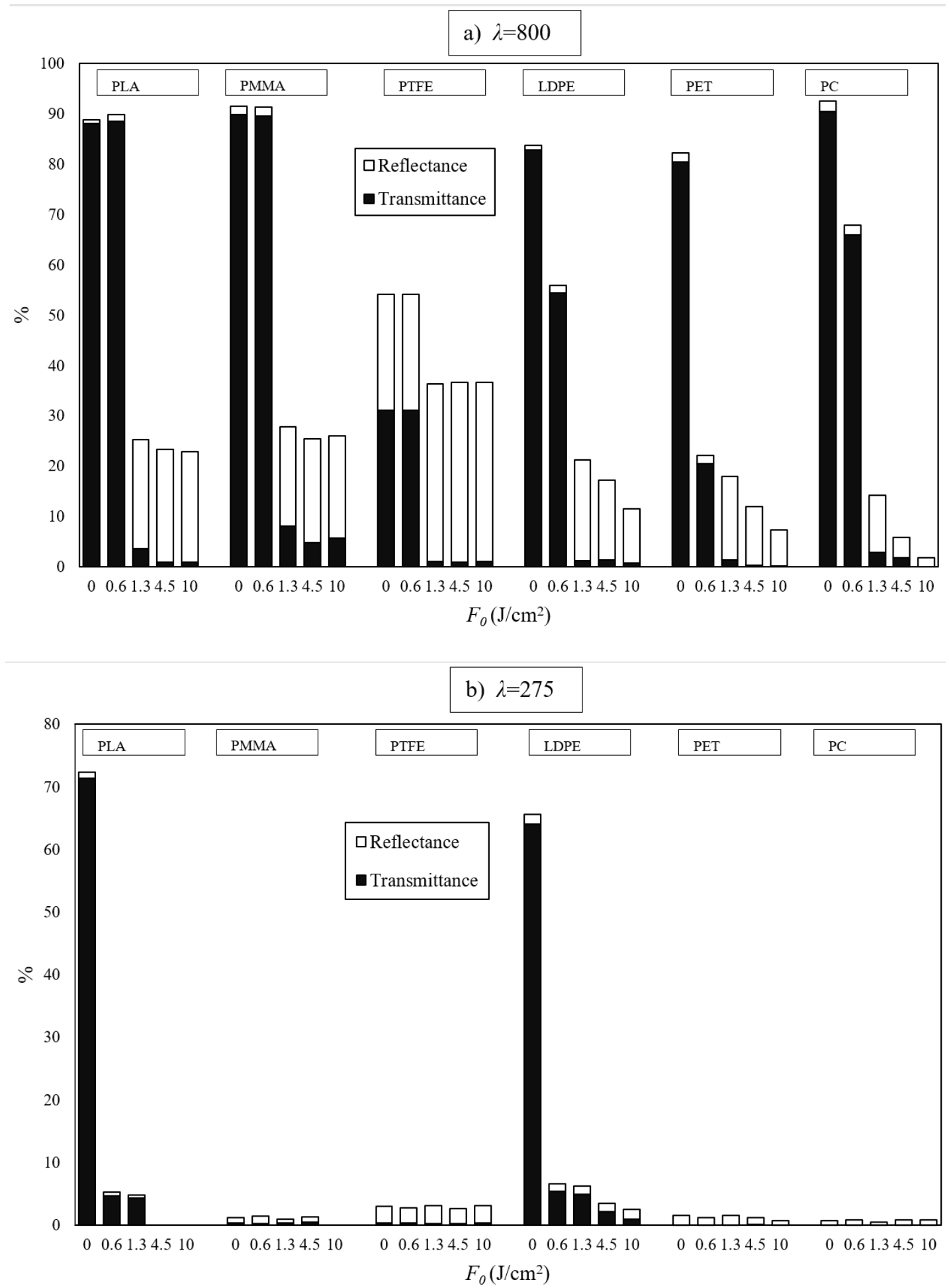

Figure 4: Transmittance and reflectance at the machining wavelength $\lambda$ for a) $\lambda=800 \mathrm{~nm}$ and b) $\lambda=275 \mathrm{~nm}$. 
In conclusion, UV-VIS data strongly suggests that a shift in optical properties, namely an increase in absorption, occurs during machining This is expected to have an impact on the threshold fluence, the magnitude of which was recently observed to have a significant effect on microstructure formation [16]. Accordingly, we determined the single pulse threshold fluences and incubation coefficients of all materials at both wavelengths using the method described in section 2.2 .

\subsection{Single pulse threshold fluence and incubation coefficient:}

Figure 5 exemplarily describes the evolution of ablation depth at different pulse numbers and fluences for PLA at $\lambda=800 \mathrm{~nm}$.
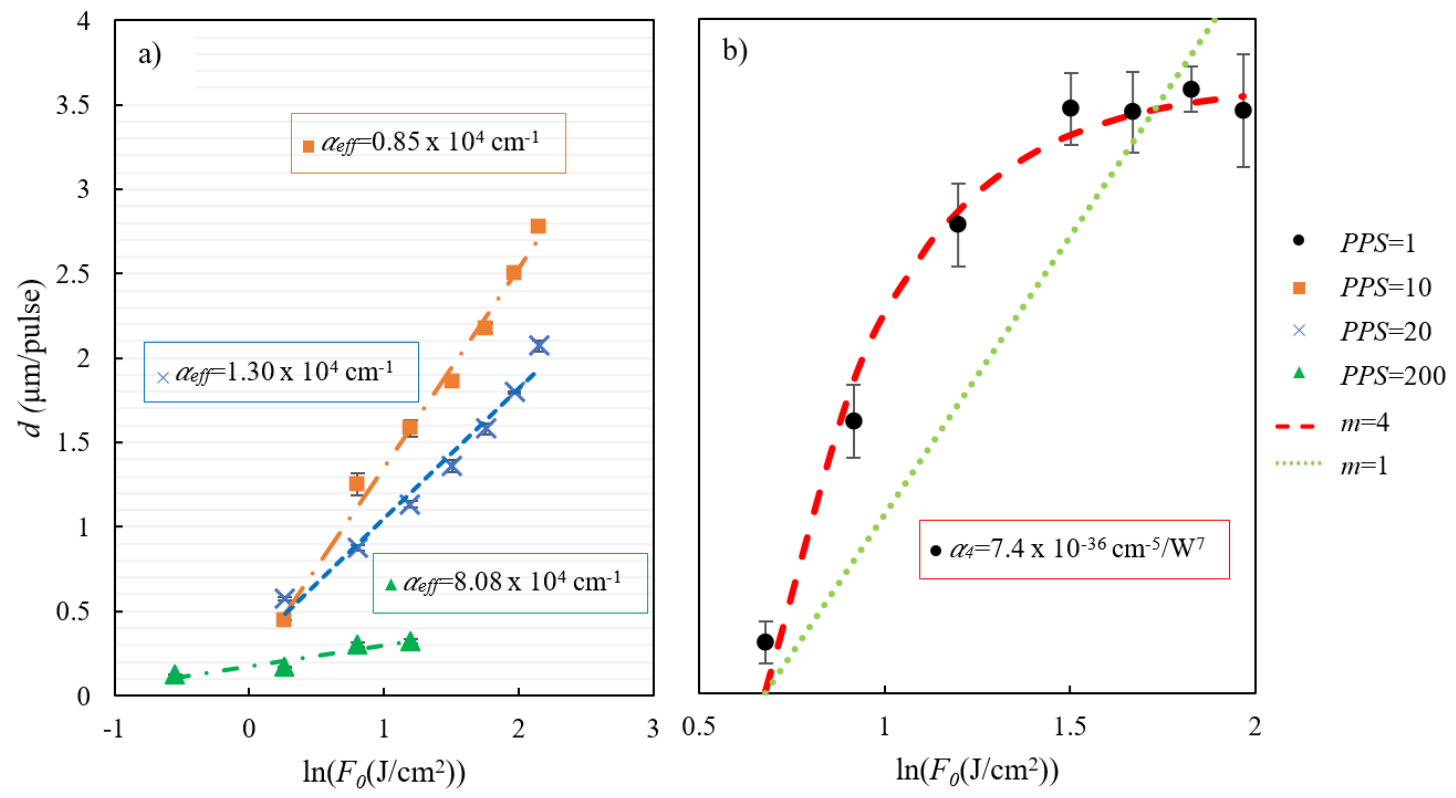

Figure 5: Ablation depth per pulse for PLA at $\lambda=800 \mathrm{~nm}$ with corresponding $\alpha$ at each PPS for a) multi-pulse ablation and b) singlepulse ablation with a comparison between the best fits of the linear and four-photon absorption models. Error bars represent the standard deviation of three measurements.

As can be seen in Figure 5a, the depth-per-pulse data for multi-pulse ablation is in good agreement with equation 3. Furthermore, a clear decrease in slope is observed with increasing PPS numbers, which corresponds to an increase in the effective absorption coefficient. The same 
behavior was observed for all six polymers. This is further indication that, in addition to lowering the threshold fluence, incubation effects related to machining at higher PPS numbers alter a polymer's optical properties. Such behavior has been reported for fs laser ablation of semiconductors such as fused silica [31] and polymers such as PMMA [32]. Incubation effects are believed to originate from physical and/or chemical defects that are generated by laser irradiation [1]. The exact nature and formation mechanism of these defects is still under debate and several mechanisms have been proposed such as the enhanced energy coupling efficiency due to laserinduced surface plasmons [33] and the accumulation of defect sites due to the excitation and generation of conduction-band electrons in dielectrics [34]. For the specific case of fs laser ablation of polymers, Baudach et al. (2001) observed that materials with ester bonds showcased higher incubation effects, which they suggested might be due to preferential bond breaking [14].

On the other hand, single-pulse ablation data did not provide a good fit for any of the transparent films as illustrated for PLA in Figure 5b. As can be seen, the ablation depth does not seem to follow the linear absorption model described by equation 3 (light green dotted line) as it seems to plateau at high fluence. This is unsurprising since this model is derived from the BeerLambert law for linearly absorbing materials. Transparent materials have a large bandgap energy that is typically larger than the energy of a photon emitted at an $800 \mathrm{~nm}$ wavelength and thus multiphoton absorption models are better adapted to describe their ablation behavior. Despite that fact, depth vs. fluence data for most polymers have been reported to follow a linear relationship of the same type as equation 3 with the non-linear optical effects being included in the coefficient $\alpha_{\text {eff }}$ [20]. This has also been observed for our multi-pulse results (Figure 5a). For the sake of comparison, the single pulse ablation data was fit to a multiphoton ablation model based on the work of Preuss et al. (1993) [35]. The model is described by the equation below:

$$
d=\frac{\tau^{m-1}}{(m-1) \alpha_{m}}\left(\frac{1}{F_{t h}^{m-1}}-\frac{1}{F_{0}^{m-1}}\right)
$$

where $m$ represents the order of photon ablation and $\alpha_{m}$ the corresponding absorption coefficient. In equation 5, all of the absorption processes of order lower than $m$ are ignored. For PLA, the best fit was obtained for a four-photon absorption model $(m=4)$. Through UV/VIS spectrometry, the cut-off wavelength $\lambda_{c}$ was measured to be $235 \mathrm{~nm}$ for PLA, which corresponds to an optical bandgap energy of $E_{g}=5.28 \mathrm{eV}$. Considering that a photon at a wavelength of $800 \mathrm{~nm}$ has a photon energy of $E_{p}=1.55 \mathrm{eV}$, then at least four photons are required to be absorbed simultaneously in order to cross the optical bandgap of the material. Thus, the use of a four-photon absorption model is justified. Table 2 summarizes the parameters obtained from the different model fits for all polymers at both $800 \mathrm{~nm}$ and $275 \mathrm{~nm}$. 
Table 2: Comparison of best fit model parameters at $\lambda=800 \mathrm{~nm}$ and $\lambda=275 \mathrm{~nm}$

\begin{tabular}{|c|c|c|c|c|c|c|c|c|c|c|c|}
\hline & \multicolumn{2}{|c|}{$\begin{array}{l}F_{t h}(\mathbf{1}) \\
\left(\mathbf{J} / \mathbf{c m}^{2}\right)\end{array}$} & \multicolumn{2}{|c|}{$\xi$} & \multicolumn{2}{|c|}{$\begin{array}{c}m \text { for } \\
P P S=1\end{array}$} & \multicolumn{2}{|c|}{$\begin{array}{l}\alpha_{m} \text { for } P P S=1 \\
\left(\mathrm{~cm}^{2 \mathrm{~m}-3} / \mathbf{W}^{\mathrm{m}-1}\right)\end{array}$} & \multicolumn{2}{|c|}{$\begin{array}{c}\alpha_{e f f} \text { for } P P S=200 \\
\left(\mathrm{~cm}^{-1}\right)\end{array}$} & \multirow[t]{2}{*}{$\lambda_{c}(\mathbf{n m})$} \\
\hline$\lambda(\mathrm{nm})$ & 800 & 275 & 800 & 275 & 800 & 275 & 800 & 275 & 800 & 275 & \\
\hline LDPE & 1.79 & 1.27 & 0.70 & 0.72 & 4 & 2 & $3.1 \times 10^{-35}$ & $1.1 \times 10^{-11}$ & $7.0 \times 10^{4}$ & $8.7 \times 10^{4}$ & 205 \\
\hline $\mathrm{PC}$ & 1.72 & 0.31 & 0.73 & 0.92 & 3 & 1 & $8.7 \times 10^{-24}$ & $7.0 \times 10^{4}$ & $4.2 \times 10^{4}$ & $7.4 \times 10^{4}$ & 281 \\
\hline PET & 2.07 & 0.30 & 0.65 & 0.87 & 3 & 1 & $9.2 \times 10^{-24}$ & $8.7 \times 10^{4}$ & $4.9 \times 10^{4}$ & $1.1 \times 10^{5}$ & 314 \\
\hline PLA & 2.08 & 1.02 & 0.61 & 0.63 & 4 & 2 & $7.4 \times 10^{-36}$ & $2.1 \times 10^{-12}$ & $8.1 \times 10^{4}$ & $8.7 \times 10^{4}$ & 235 \\
\hline PMMA & 2.51 & 0.52 & 0.71 & 0.89 & 3 & 1 & $3.6 \times 10^{-24}$ & $3.2 \times 10^{4}$ & $3.6 \times 10^{4}$ & $6.8 \times 10^{4}$ & 380 \\
\hline PTFE & 2.25 & 1.02 & 0.84 & 0.86 & 1 & 1 & $1.2 \times 10^{4}$ & $1.4 \times 10^{4}$ & $2.6 \times 10^{4}$ & $6.7 \times 10^{4}$ & NA* \\
\hline
\end{tabular}

*No $\lambda_{c}$ value is listed for PTFE since, unlike the other films, its transmittance spectrum did not exhibit a sharp increase at a certain wavelength.

Operating at $800 \mathrm{~nm}$, the only polymer that did not exhibit a multiphoton absorption behavior for single pulse ablation is PTFE (which was the only non-transparent polymer). It also displayed the lowest increase in effective absorption coefficient between $P P S=1$ and $P P S=200$ and the largest incubation coefficient. For the other five polymers, the multiphoton ablation model provided the best fit with the value of $m$ being in agreement with their respective $\lambda_{c}$ values. Therefore, the predominance of multiphoton absorption processes for single pulse ablation of transparent polymers is confirmed. However, all of them also experienced a transition to a linear absorption behavior at higher PPS accompanied by a very large increase in $\alpha_{\text {eff. }}$ The results at 275 $\mathrm{nm}$ were significantly different. First of all, all polymers underwent a large decrease in single pulse threshold fluence owing to the increased photon energies at this wavelength. PLA and LDPE, who have a $\lambda_{c}$ that was even lower than $275 \mathrm{~nm}$, still displayed a multiphoton absorption behavior with very low single pulse absorption. They also experienced a very slight increase in incubation coefficient that, nevertheless, remained relatively low. On the other hand, data for PC, PET, and PMMA followed the linear absorption model even for single-pulse ablation. Consequently, they displayed high absorption even at lower pulse numbers and no transition in their absorption behavior at higher pulse numbers. Coincidentally, they all experienced a large increase in incubation coefficient which means they were much less affected by increasing pulse numbers at $275 \mathrm{~nm}$ than at $800 \mathrm{~nm}$. In conclusion, this is in agreement with the previous UV/VIS results and clearly indicates that a transition from a multiphoton to a linear absorption behavior is the main contributor to incubation effects in polymers. Therefore, the multiphoton absorption model is only 
valid for single or low pulse numbers since, as soon as some microstructure is formed, it will linearly absorb the subsequent pulses.

Using this data, the threshold fluence at operating conditions $(P P S=55)$ for each material was calculated at both wavelengths using equation 4 and summarized in Table 3.

Table 3: Threshold fluences at operating conditions.

\begin{tabular}{|c|c|c|}
\hline Material & $F_{t h}(55)\left(J / c^{2}\right)$ at $\lambda=800 \mathrm{~nm}$ & $F_{t h}(55)\left(J / \mathrm{cm}^{2}\right)$ at $\lambda=275 \mathrm{~nm}$ \\
\hline LDPE & 0.54 & 0.41 \\
\hline $\mathrm{PC}$ & 0.59 & 0.23 \\
\hline PET & 0.51 & 0.18 \\
\hline PLA & 0.45 & 0.23 \\
\hline PMMA & 0.79 & 0.34 \\
\hline PTFE & 1.18 & 0.58 \\
\hline
\end{tabular}

By looking at the threshold fluence values for operation at $275 \mathrm{~nm}$, it can be seen that LDPE and PTFE, which were the only two polymers to retain the porous structure at this wavelength (as seen from Figure 2), had the largest $F_{\text {th }}$ values. PMMA had the third largest threshold value and exhibited a small degree of porosity as well. On the other hand, all other materials exhibited significantly lower threshold values. Therefore, as our previous work suggests, porous laser-induced structure formation appears to be linked to the ablation threshold at the operating PPS number. PMMA is the polymer that is most commonly reported as observing laserinduced porosity. As first suggested by Efthimiopoulos et al. (2000), this type of morphology could be attributed to an explosive boiling mechanism [36]. In laser ablation, the incoming energy is absorbed on a ps time scale and a layer of material is removed by ionization. Depending on the pulse duration, the underlying layers get heated to certain extent and form a hydrodynamic melt layer that re-solidifies in the order of nanoseconds [37]. For ultrashort laser machining, rapid adiabatic expansion and quasi-isochoric heating can lead to superheating of the melt volume and homogeneous bubble formation which is termed as explosive boiling [38]. Baudach et al, (2000) [9] suggest that the porosity observed at the bottom and sidewalls of fs laser ablated PMMA 
features are due to trapped bubbles in the re-solidifying melt layer. This notion was reinforced by numerical simulations performed by Guay et al. (2013) [39]. Assuming that our observed laserinduced microstructures are formed through a similar mechanism, the dependence on the threshold fluence at operating conditions might then be explained. As observed in Figure 6, during fs laser machining, the portion of the fluence profile of the incoming Gaussian pulses that is above the threshold fluence value of the polymer will lead to the ablation of the outermost layers. The underlying layers, that are only exposed to fluences up to $F_{t h}$, will undergo melting. The degree of superheating of this melt layer depends on the magnitude of the fluence to which it is exposed. It is therefore also dependent on $F_{t h}$, which is equivalent to the highest fluence a material can withstand without undergoing ionization. Therefore, the higher $F_{t h}$ is, the larger the degree of superheating of the melt layer. If the critical temperature for explosive boiling $T_{c}$ is not reached, which is the case for polymers with a low $F_{t h}$ value, bubble formation will therefore not occur. Finally, Figure 7 represents the microstructure observed on LDPE for $\lambda=275 \mathrm{~nm}$ at a fluence just above the threshold value $\left(F_{0}=0.45 \mathrm{~J} / \mathrm{cm}^{2}\right)$. As can be seen, the outermost layer is getting peeled off due to the emerging pores. This is in line with the explosive boiling mechanism as the rising bubbles would coalesce near the outermost re-solidifying layer and cause compressive stresses leading to material ejection through spallation [40].
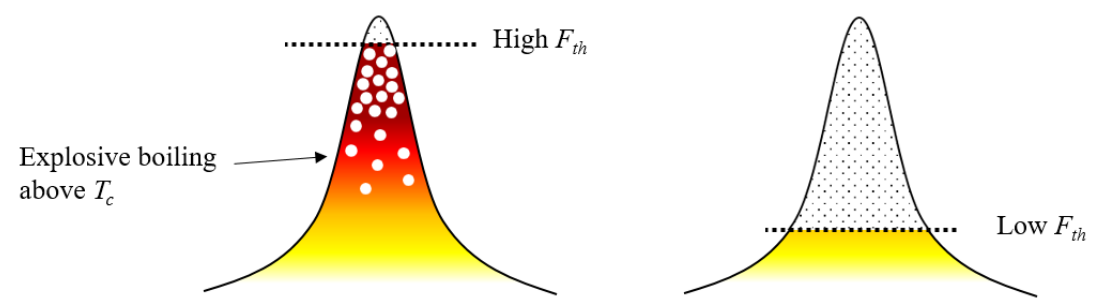

Ionized material

Melt at a temperature higher than $T_{c}$ Melt at a temperature lower than $T_{c}$

Figure 6: Effect of threshold fluence on temperature of the re-solidifying melt layer 


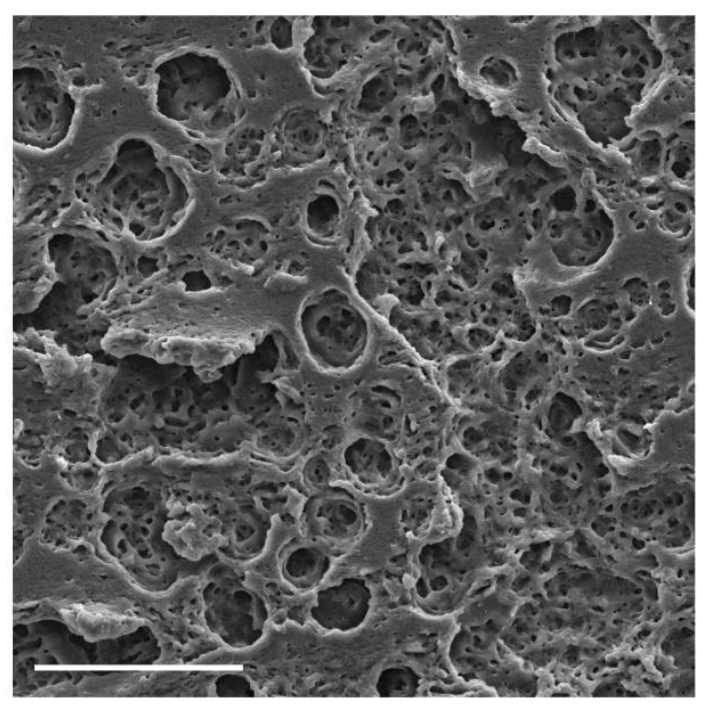

Figure 7: Spallation on LDPE machined at $F_{0}=0.45 \mathrm{~J} / \mathrm{cm}^{2}$ and $\lambda=275 \mathrm{~nm}$. Scale bar represents $10 \mu \mathrm{m}$.

In summary, the fits of the different absorption models confirm the large changes in optical properties that were measured by UV/VIS spectrometry. These changes heavily affect polymer machinability and the type of laser-induced structures that can appear on their surface. While the formation of a re-solidified melt layer and the accompanying increase in roughness can partly explain the observed increases in absorption and reflectance, it does not explain the surface darkening observed on LDPE, PC, and PET. Thus, as a final step, XPS analysis was performed on all samples to check if initial surface chemistry and photochemical reactions contribute to any of the aforementioned phenomena.

\subsection{Chemical characterization:}

The chemical composition and bonding structure were measured for all structures at both wavelengths. The elemental composition of all samples initially only contains carbon, oxygen, and hydrogen with the exception of PTFE, which is composed of a 2:1 fluorine-to-carbon mixture. Figure 8 presents the changes in composition as a function of fluence by displaying the atomic percentage of oxygen. These measurements omit hydrogen since it is undetectable by XPS analysis. On the other hand, Figure 9 summarizes the evolution of the bonding structure behavior of all six materials by comparing the high resolution $\mathrm{C} 1 \mathrm{~s}$ spectra for the non-modified material and a patch machined at high fluence $\left(F_{0}=7.2 \mathrm{~J} / \mathrm{cm}^{2}\right)$ at each wavelength. Lower values were 
chosen for PLA $\left(F_{0}=3.3 \mathrm{~J} / \mathrm{cm}^{2}\right)$ and PMMA $\left(F_{0}=5.8 \mathrm{~J} / \mathrm{cm}^{2}\right)$ because they underwent full ablation through the film at higher fluences.
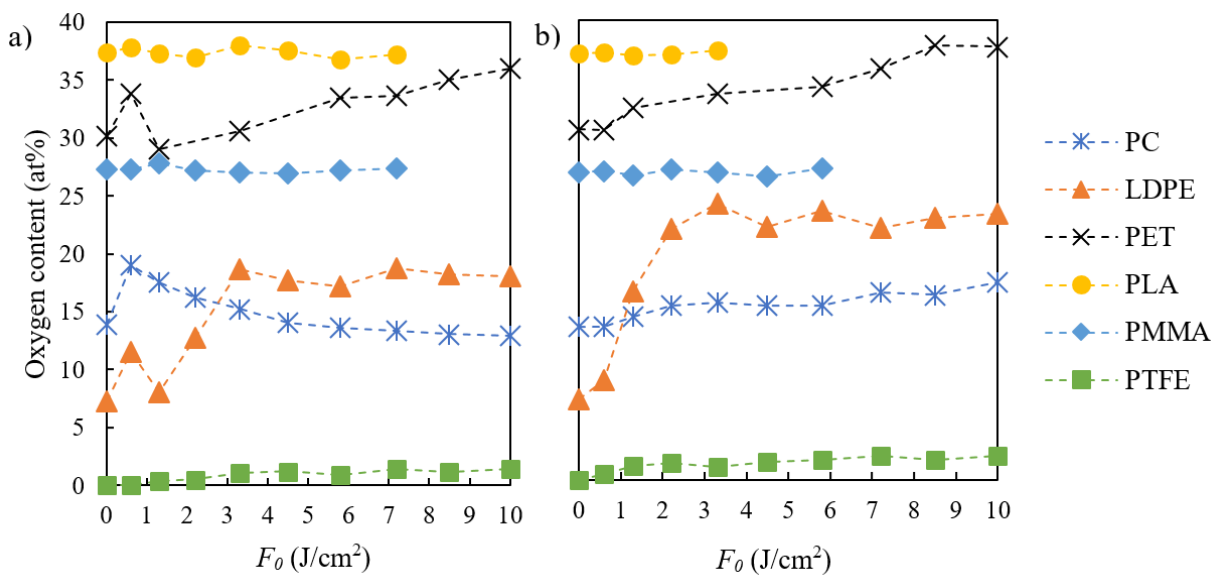

Figure 8: Evolution of oxygen content as a function of fluence for machining at a) $\lambda=800 \mathrm{~nm}$ and b) $\lambda=275 \mathrm{~nm}$. Fewer data points were collected for PMMA and PLA because, at higher fluence, the entirety of the film was ablated.

Considering Figures 8 and 9, four out of the six polymers seem to undergo photochemical reactions during machining. Indeed, for LDPE, PC, PET, and, to a lesser extent, PTFE, a dependence of surface oxygen content on fluence can be observed at both wavelengths (Figure 8). Firstly, we note that, with the exception of PTFE, this group of polymers experiences an increase in oxygen content below ablation threshold $\left(F_{0}=0.6 \mathrm{~J} / \mathrm{cm}^{2}\right)$ at $\lambda=800 \mathrm{~nm}$. Looking back at Figure 1 , this coincides with a color change even if no microstructure is formed. However, the oxygen content drops down close to its original value once ablation is reached $\left(F_{0}=1.3 \mathrm{~J} / \mathrm{cm}^{2}\right)$ which means that the oxidized layer was removed as part of the ablation process leaving behind the porous structure. Beyond the ablation threshold $\left(F_{0}>1.3 \mathrm{~J} / \mathrm{cm}^{2}\right)$, all four polymers once again experience an increase of surface oxygen content and increasing surface darkening with fluence at both wavelengths. The only exception being PC at $\lambda=800 \mathrm{~nm}$ that actually experiences an overall decrease in oxygen which might be due to the observed degradation of its microstructure (Figure 1). The fact that PTFE did not go through similar changes in color could be due to the fact that its chemical structure changed only slightly (Figure 8). In conclusion, color change after fs laser micromachining seems to be related to surface oxidization. 


\section{Pristine}

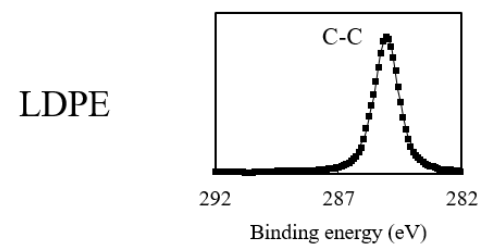

PTFE

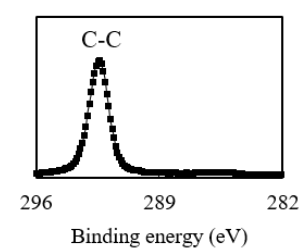

PET

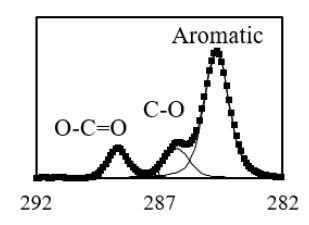

PC
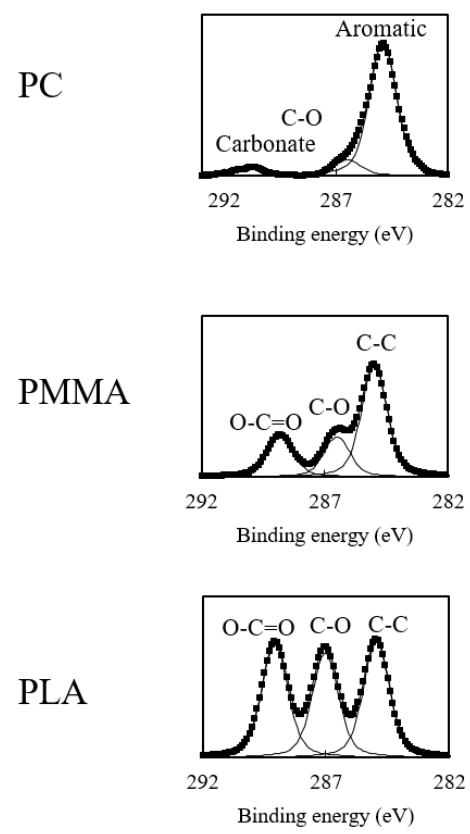
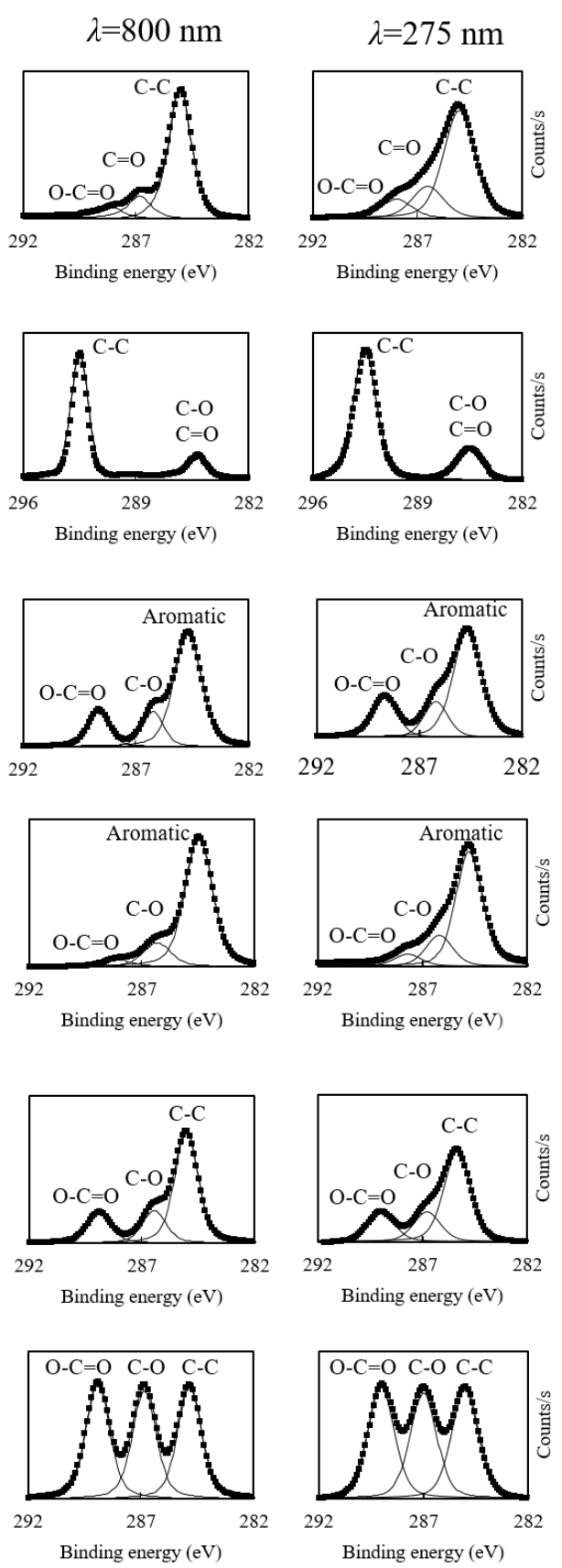

Figure 9: High resolution C1s spectra for non-modified polymers (left) and machined at high fluence $\left(F_{0}=7.2 \mathrm{~J} / \mathrm{cm}^{2}\right)$ for $\lambda=800 \mathrm{~nm}$ (middle) and $\lambda=275 \mathrm{~nm}$ (right). Lower values were chosen for PLA $\left(\mathrm{F}_{0}=3.3 \mathrm{~J} / \mathrm{cm}^{2}\right)$ and PMMA $\left(\mathrm{F}_{0}=5.8 \mathrm{~J} / \mathrm{cm}^{2}\right)$ because they underwent full ablation through the film at higher fluences. Both measured data (square dots) and individual labeled peak fits (solid lines) are shown. 
Further insight into the mechanisms behind these chemical changes can be obtained by analyzing the changes in bonding structure after machining for each of the concerned polymers (Figure 9). For LDPE, PC, PET, and PTFE, the observed increase in oxygen content (Figure 8) is reflected in their high resolution $\mathrm{C} 1 \mathrm{~s}$ scans. At $\lambda=800 \mathrm{~nm}$, for all four polymers, either the relative amount of oxidized carbon increased at the expense of hydrocarbon bonds (PET), or new carbon oxygen peaks have appeared (LDPE, PC, and PTFE). In addition, the same type of oxidation was observed for $\lambda=275 \mathrm{~nm}$. However, it was much more pronounced at this wavelength as the oxidation peaks were significantly larger. Indeed, the proportion of carbon-oxygen bonds with respect to total carbon bonds (calculated from the area of the fitted peaks) increased from $28 \%$ to $37 \%$ for LDPE, $8 \%$ to $10 \%$ for PTFE, $21 \%$ to $26 \%$ for PET, and from $20 \%$ to $26 \%$ for PC. The dependence of the magnitude of the oxidation process on wavelength can be explained by considering photo-oxidative mechanisms prominent under ultraviolet irradiation.

Indeed, polymers are known to undergo photo-oxidative degradation when subjected to a UV light source which is generally accompanied by the appearance of a yellow color. The general mechanism of these reactions has been widely studied and they are known to produce several oxygen containing functional groups such as aldehydes, ketones and carboxylic acids [41]. Oxidation is typically initiated by free radical formation and reaction with atmospheric oxygen. Many internal chromophoric groups can act as initiator sites such as impurities, unsaturated carbon, tertiary carbon, and carbonyl groups among others [42]. The changes in bonding structure observed in Figure 9 are consistent with the established mechanisms for LDPE [43] and PET [44]. In the case of the former, initiation occurs by peroxy radical formation and subsequent chain scission and results in both aldehyde and carboxylic functional group formation. On the other hand, PET undergoes hydroperoxide formation and subsequent chain scission and results in an increase in carboxylic group formation. In the case of PC, the behavior at $\lambda=275 \mathrm{~nm}$ can be explained by a photooxidative process via hydroperoxide formation and reaction with oxygen [45]. However, this does not explain the net decrease in oxygen at high fluence (Figure 8) and the decrease of the carbonate peak when irradiating PC at $\lambda=800 \mathrm{~nm}$ (Figure 9) that are concurrent with the appearance of a black color (Figure 1). One reaction that could explain this is the hydrolysis of the carbonate group that is ejected as carbon dioxide by reaction with atmospheric water vapor. The detailed mechanism of PC's reaction with water can be found in Pan et al.'s (2009) study [46]. In contrast to these three polymers, PTFE is not known to typically undergo photo-oxidation. However, Golub 
et al. (1991) observed a peak similar to the one in Figure 9 on a PTFE surface treated with oxygen plasma [47]. The authors attributed this peak to oxidized carbon and hydrocarbon contamination. In summary, the high resolution XPS scans suggest that photo-oxidative mechanisms are behind the increase in oxygen content observed after machining.

Since photo-oxidation typically occurs on polymers during UV irradiation due to their high absorption at these wavelengths, it is unsurprising that such reactions were observed to take place when operating at $\lambda=275 \mathrm{~nm}$. However, the same type of reaction also occurred at $\lambda=800 \mathrm{~nm}$, which demonstrates that the microstructure formed beyond the ablation threshold is absorbent to this wavelength unlike the base material. This once again reinforces the notion of a large increase in absorption after machining as already demonstrated in the UV-VIS analysis (Figure 4) and the absorption model fits (Table 2).

Finally, the two remaining polymers (PLA and PMMA) showed no change in composition or bonding structure as a function of fluence. For both materials, the peaks retain the same proportions throughout the tested machining conditions at both wavelengths. This behavior can be explained for PMMA since it is known for its outstanding resistance to photo-oxidation as it does not contain internal chromophoric groups that could initiate free radical formation [48]. Furthermore, it is generally produced at extremely high purities which rules out any impurityinduced chromophores. On the other hand, PLA's lack of reactivity can be attributed to its extreme susceptibility to thermal degradation [49]. Jia et al. (2015) have similarly shown that femtosecond laser micromachining of PLA is a non-oxidative process as thermal degradation was observed instead [50].

In conclusion, XPS analysis has shown that polymers that are susceptible to photooxidation will experience an increase in surface oxygen content after machining. This leads to a darkening of the surface and increase in absorption that increases with operating fluence. In addition, these reactions are not limited to UV ablation as the same type of reaction is observed for $\lambda=800 \mathrm{~nm}$ which we attribute to the much larger absorption of the induced microstructure with respect to this wavelength when compared the non-modified material. Therefore, irrespective of the machining wavelength, photo-oxidative reactions need to be taken into account when considering the final properties of laser induced microstructures that appear after femtosecond laser micromachining of polymers. 


\section{CONCLUSION}

In this study, we optically and chemically characterized the femtosecond laser-induced microstructures that appear on several polymers at different operating fluences and at two separate wavelengths. At $\lambda=800 \mathrm{~nm}$, all polymers formed a porous structure. In addition, LDPE, PET, and PC showed a darkening of their surface at higher fluences. At $\lambda=275 \mathrm{~nm}$, only PTFE and LDPE retained the porous structure, while the other polymers mostly experienced melt formation. This was attributed to the threshold fluence of these two polymers that was still high enough at this wavelength for explosive boiling to occur. The magnitude of the threshold fluence was further determined to be dependent on the changes in optical properties that occur during machining. UVVIS spectrometry showed that an increase in absorption and reflectance occurred when the laserinduced microstructures were formed. In addition, LDPE, PET and PC, were observed to be increasingly absorbent as fluence increased. Fitting of different absorption models confirmed the observed increases in absorption. Furthermore, it revealed that a transition from a multiphoton absorption behavior during single pulse ablation to linear absorption at higher pulse numbers was occurring for transparent polymers. This shift was identified as being the main contributor to incubation effects. It is therefore crucial in determining the threshold fluence when operating at high pulse numbers. Finally, XPS analysis revealed that photo-oxidative mechanisms are behind the darkening and continuous increase in absorption observed on LDPE, PC, and PET. In conclusion, we identified the main parameters that lead to porous structure formation on polymer surfaces as well as the optical and chemical processes that affect them.

\section{Author contributions}

Y. Assaf wrote the manuscript, designed and conducted all the experiments, and all the analysis. A.-M. Kietzig supervised the work and edited the manuscript. 


\section{Funding information}

This work was supported by the National Sciences and Research Council of Canada (NSERC).

\section{Conflicts of interest}

The authors declare no conflict of interest.

\section{References}

[1] D. Bauerle, Ultrashort-Pulse Laser Ablation, Laser Processing and Chemistry, Springer Berlin Heidelberg, Berlin, Heidelberg, 2011, pp. 279-313.

[2] M.D. Shirk, P.A. Molian, A review of ultrashort pulsed laser ablation of materials, Journal of Laser Applications 10(1) (1998) 18-28.

[3] A. del Campo, E. Arzt, Fabrication Approaches for Generating Complex Micro- and Nanopatterns on Polymeric Surfaces, Chemical Reviews 108(3) (2008) 911-945.

[4] K.M.T. Ahmmed, C. Grambow, A.-M. Kietzig, Fabrication of Micro/Nano Structures on Metals by Femtosecond Laser Micromachining, Micromachines 5(4) (2014) 1219.

[5] A.Y. Vorobyev, C. Guo, Colorizing metals with femtosecond laser pulses, Applied Physics Letters 92(4) (2008) 041914.

[6] B. Wu, M. Zhou, J. Li, X. Ye, G. Li, L. Cai, Superhydrophobic surfaces fabricated by microstructuring of stainless steel using a femtosecond laser, Applied Surface Science 256(1) (2009) 61-66.

[7] A.-M. Kietzig, S.G. Hatzikiriakos, P. Englezos, Patterned Superhydrophobic Metallic Surfaces, Langmuir 25(8) (2009) 4821-4827.

[8] A.Y. Vorobyev, C. Guo, Multifunctional surfaces produced by femtosecond laser pulses, Journal of Applied Physics 117(3) (2015) 033103.

[9] S. Baudach, J. Bonse, J. Kruger, W. Kautek, Ultrashort pulse laser ablation of polycarbonate and polymethylmethacrylate, Applied Surface Science 154-155(Supplement C) (2000) 555-560.

[10] S. Yada, M. Terakawa, Femtosecond laser induced periodic surface structure on poly-L-lactic acid, Optics Express 23(5) (2015) 5694-5703.

[11] J. Heitz, B. Reisinger, M. Fahrner, C. Romanin, J. Siegel, V. Svorcik, Laser-induced periodic surface structures (LIPSS) on polymer surfaces, 2012 14th International Conference on Transparent Optical Networks (ICTON), 2012, pp. 1-4.

[12] E. Rebollar, J.R. Vazquez de Aldana, I. Martin-Fabiani, M. Hernandez, D.R. Rueda, T.A. Ezquerra, C. Domingo, P. Moreno, M. Castillejo, Assessment of femtosecond laser induced periodic surface structures on polymer films, Physical Chemistry Chemical Physics 15(27) (2013) 11287-11298.

[13] F. Baset, A. Villafranca, J.-M. Guay, R. Bhardwaj, Femtosecond laser induced porosity in poly-methyl methacrylate, Applied Surface Science 282(Supplement C) (2013) 729-734.

[14] S. Baudach, J. Kruger, W. Kautek, Femtosecond Laser Processing of Soft Materials, The Review of Laser Engineering 29(11) (2001) 705-709.

[15] F. Liang, J. Lehr, L. Danielczak, R. Leask, A.-M. Kietzig, Robust Non-Wetting PTFE Surfaces by Femtosecond Laser Machining, International Journal of Molecular Sciences 15(8) (2014) 13681. 
[16] Y. Assaf, A.-M. Kietzig, Formation of porous networks on polymeric surfaces by femtosecond laser micromachining, SPIE LASE, SPIE, 2017, p. 11.

[17] A.W. Martinez, E.L. Chaikof, Microfabrication and nanotechnology in stent design, Wiley Interdisciplinary Reviews: Nanomedicine and Nanobiotechnology 3(3) (2011) 256-268.

[18] S.T. Rashid, H.J. Salacinski, B.J. Fuller, G. Hamilton, A.M. Seifalian, Engineering of bypass conduits to improve patency, Cell Proliferation 37(5) (2004) 351-366.

[19] S. Kuper, M. Stuke, UV-excimer-laser ablation of polymethylmethacrylate at $248 \mathrm{~nm}$ : Characterization of incubation sites with Fourier transform IR- and UV-Spectroscopy, Applied Physics A 49(2) (1989) 211215.

[20] J. Kruger, W. Kautek, Ultrashort Pulse Laser Interaction with Dielectrics and Polymers, in: T.K. Lippert (Ed.), Polymers and Light, Springer Berlin Heidelberg, Berlin, Heidelberg, 2004, pp. 247-290.

[21] M. Okoshi, N. Inoue, Laser ablation of polymers using $395 \mathrm{~nm}$ and $790 \mathrm{~nm}$ femtosecond lasers, Applied Physics A 79(4) (2004) 841-844.

[22] O. Masayuki, I. Narumi, Femtosecond Laser Ablation of Polyethylene, Japanese Journal of Applied Physics 42(1A) (2003) L36.

[23] T. Lippert, J.T. Dickinson, S.C. Langford, H. Furutani, H. Fukumura, H. Masuhara, T. Kunz, A. Wokaun, Photopolymers designed for laser ablation - photochemical ablation mechanism, Applied Surface Science 127-129(Supplement C) (1998) 117-121.

[24] R. Srinivasan, R.R. Hall, W.D. Loehle, W.D. Wilson, D.C. Allbee, Chemical transformations of the polyimide Kapton brought about by ultraviolet laser radiation, Journal of Applied Physics 78(8) (1995) 4881-4887.

[25] S. Baudach, J. Bonse, W. Kautek, Ablation experiments on polyimide with femtosecond laser pulses, Applied Physics A 69(1) (1999) S395-S398.

[26] J.M. Liu, Simple technique for measurements of pulsed Gaussian-beam spot sizes, Optics Letters 7(5) (1982) 196-198.

[27] Y. Jee, M.F. Becker, R.M. Walser, Laser-induced damage on single-crystal metal surfaces, Journal of the Optical Society of America B 5(3) (1988) 648-659.

[28] G. Beamson, D. Briggs, High Resolution XPS of Organic Polymers: The Scienta ESCA300 Database, Journal of Chemical Education 70(1) (1993) A25.

[29] A. Naghilou, O. Armbruster, M. Kitzler, W. Kautek, Merging Spot Size and Pulse Number Dependence of Femtosecond Laser Ablation Thresholds: Modeling and Demonstration with High Impact Polystyrene, The Journal of Physical Chemistry C 119(40) (2015) 22992-22998.

[30] L.G. DeShazer, B.E. Newnam, K.M. Leung, Role of coating defects in laser- induced damage to dielectric thin films, Applied Physics Letters 23(11) (1973) 607-609.

[31] M. Lenzner, J. Kruger, W. Kautek, F. Krausz, Incubation of laser ablation in fused silica with 5-fs pulses, Applied Physics A 69(4) (1999) 465-466.

[32] S. Kuper, M. Stuke, Femtosecond uv excimer laser ablation, Applied Physics B 44(4) (1987) 199-204.

[33] J. Byskov-Nielsen, J.-M. Savolainen, M.S. Christensen, P. Balling, Ultra-short pulse laser ablation of metals: threshold fluence, incubation coefficient and ablation rates, Applied Physics A 101(1) (2010) 97101.

[34] A. Rosenfeld, M. Lorenz, R. Stoian, D. Ashkenasi, Ultrashort-laser-pulse damage threshold of transparent materials and the role of incubation, Applied Physics A 69(1) (1999) S373-S376.

[35] S. Preuss, M. Spath, Y. Zhang, M. Stuke, Time resolved dynamics of subpicosecond laser ablation, Applied Physics Letters 62(23) (1993) 3049-3051.

[36] T. Efthimiopoulos, C. Kiagias, G. Heliotis, E. Helidonis, Evidence of volume bubble creation during laser ablation of PMMA organic polymer, Canadian Journal of Physics 78(5-6) (2000) 509-519.

[37] S.I. Anisimov, N.A. Inogamov, A.M. Oparin, B. Rethfeld, T. Yabe, M. Ogawa, V.E. Fortov, Pulsed laser evaporation: equation-of-state effects, Applied Physics A 69(6) (1999) 617-620.

[38] A. Miotello, R. Kelly, Critical assessment of thermal models for laser sputtering at high fluences, Applied Physics Letters 67(24) (1995) 3535-3537. 
[39] J.M. Guay, A. Villafranca, F. Baset, K. Popov, L. Ramunno, V.R. Bhardwaj, Polarization-dependent femtosecond laser ablation of poly-methyl methacrylate, New Journal of Physics 14(8) (2012) 085010.

[40] D. Perez, L.J. Lewis, Molecular-dynamics study of ablation of solids under femtosecond laser pulses, Physical Review B 67(18) (2003) 184102.

[41] J.F. Rabek, Photochemical aspects of degradation of polymers, Polymer Photodegradation: Mechanisms and experimental methods, Springer Netherlands, Dordrecht, 1995, pp. 24-66.

[42] E. Yousif, R. Haddad, Photodegradation and photostabilization of polymers, especially polystyrene: review, SpringerPlus 2(1) (2013) 398.

[43] M. Iring, F. Tudos, Thermal oxidation of polyethylene and polypropylene: Effects of chemical structure and reaction conditions on the oxidation process, Progress in Polymer Science 15(2) (1990) 217262.

[44] M. Day, D.M. Wiles, Photochemical degradation of poly(ethylene terephthalate). III. Determination of decomposition products and reaction mechanism, Journal of Applied Polymer Science 16(1) (1972) 203215.

[45] A. Factor, W.V. Ligon, R.J. May, The role of oxygen in the photoaging of bisphenol A polycarbonate. 2. GC/GC/high-resolution MS analysis of Florida-weathered polycarbonate, Macromolecules 20(10) (1987) 2461-2468.

[46] Z. Pan, I.M. Chou, R.C. Burruss, Hydrolysis of polycarbonate in sub-critical water in fused silica capillary reactor with in situRaman spectroscopy, Green Chemistry 11(8) (2009) 1105-1107.

[47] M.A. Golub, T. Wydeven, R.D. Cormia, ESCA study of the effect of hydrocarbon contamination on poly(tetrafluoroethylene) exposed to atomic oxygen plasma, Langmuir 7(5) (1991) 1026-1028.

[48] D.C. Wright, Failure of Plastics and Rubber Products: Causes, Effects and Case Studies Involving Degradation, Rapra Technology2001.

[49] I.C. McNeill, H.A. Leiper, Degradation studies of some polyesters and polycarbonates-1. Polylactide: General features of the degradation under programmed heating conditions, Polymer Degradation and Stability 11(3) (1985) 267-285.

[50] W. Jia, Y. Luo, J. Yu, B. Liu, M. Hu, L. Chai, C. Wang, Effects of high-repetition-rate femtosecond laser micromachining on the physical and chemical properties of polylactide (PLA), Optics Express 23(21) (2015) 26932-26939. 